\title{
Des ressources oléagineuses variées potentiellement au service du développement de la chimie verte
}

\author{
André MERRIEN \\ Patrick CARRE \\ Alain QUINSAC \\ CETIOM, \\ 270 Ave de la Pomme de Pin, \\ 45160 Ardon \\ $<$ merrien@cetiom.fr>
}

\begin{abstract}
The oleaginous plants are invaluable reservoirs with food use, but also industrial. In the current context of environmental protection, green oilseed based chemistery finds a revival. Bibliographical data are rather rich in experiments of introduction of new oleaginous cultures as sources of oil and fatty acids for that purpose. This note attempts to draw up an inventory.
\end{abstract}

Key words: oleaginous plants, lubricating, vegetal based chemistery, cosmetic, biofuels, plastic
Les plantes oléagineuses sont de précieux réservoirs $d^{\prime}$ acides gras à usage alimentaire mais aussi industriel. Face au défi de l'environnement, la chimie verte trouve aujourd'hui un écho particulier et les cultures oléagineuses représentent des candidates potentielles pour de nombreuses applications. L'oléochimie verte (hors saponification et biodiesel) représente déjà $7 \%$ du marché global des huiles végétales au niveau mondial. Leurs caractères renouvelable, non toxique et dégradable en font des productions intéressantes. D'autant qu'au-delà de quelques grandes espèces bien connues, de nombreuses autres cultures peuvent trouver un intérêt, pour peu que leur domestication puisse progresser. Beaucoup d'entre elles ne sont cependant pas directement adaptées à nos climats et nécessitent encore des efforts d'amélioration (teneur en huile, rendement, maîtrise technique) pour les rendre compétitives.

Outre-atlantique, des travaux de recherche sont menés sur plusieurs espèces, dont I'huile présente un profil spécifique en acides gras : jojoba, crambe d'Abyssinie, lesquerella, vernonia, cuphea, limnanthes.

En Europe, et à cette fin d'usage dédié à la chimie verte, plusieurs oléagineux à profil d'acides gras particulier ont commencé à percer : le colza érucique, le colza bas linoléique ou colza oléique, le lin oléagineux, le tournesol oléique et peut être demain le tournesol stéarique. La composition en acides gras de leurs huiles offre des débouchés multiples: biocarburants, lubrifiants, détergents, plastiques, peintures, produits cosmétiques et pharmaceutiques (Evrard, 1993).

II y a quelques années, le CETIOM a réalisé de nombreux travaux sur le potentiel de ces "cultures nouvelles" (CETIOM, 1994); citons par exemple, le ricin, la cameline, I'onagre, la bourrache, le carthame. À ce jour, peu d'entre elles ont réellement trouvé des applications significatives. Le contexte actuel d'une agriculture durable et respectueuse de l'environnement et de nouveaux marchés générés par la chimie verte mérite de revoir la question.

\section{Une diversité de cultures pour des applications diverses}

Les tableaux 1 et 2 synthétisent les données disponibles tant au niveau de la richesse en huile des graines que de la composition de cette huile en acides gras d'intérêt. Les usages sont mentionnés à titre d'applications plus souvent potentielles que correspondant à des parts de marché significatives à ce jour.

\section{Freins et leviers pour leur développement}

Parmi les cultures dédiées à ce jour à la chimie verte, le tournesol oléique est sans nul doute la plus avancée mais aussi la mieux adaptée à nos systèmes de production. Elle couvre aujourd'hui environ $50 \%$ de la sole de tournesol en France (soit environ $350000 \mathrm{ha}$ ) et son usage est principalement dédié à l'alimentaire, avec une petite part aux biocarburants. La faible insaturation de l'acide oléique confère à l'huile des propriétés de fluidité et de stabilité intéressantes pour le développement des lubrifiants (Mendoza et al., 2011). Les filières à très hautes teneurs en acide oléique (>90\%) alimentent aujourd'hui le marché de la cosmétique, des lessives et de la plasturgie.

Des variétés de tournesol stéarique sont aujourd'hui en pépinière chez les sélectionneurs. Le profil d'acides gras de ces huiles offre des teneurs en C18:0 proche de $20 \%$. II pourrait alors constituer une alternative aux huiles de palme en matière de cosmétique.

Pour citer cet article : Merrien A, Carre P, Quinsac A. Des ressources oléagineuses variées potentiellement au service du développement de la chimie verte. OCL $2012 ; 19(1)$ : 6-9. doi : 10.1684/ocl.2012.0425 
Tableau 1. Acides gras d'intérêt et usages pour quelques cultures oléagineuses cultivées (Adapté d'après Van Soest, 1993).

\begin{tabular}{|c|c|c|c|}
\hline Plante & $\begin{array}{l}\text { Teneur en huile } \\
(\%)\end{array}$ & $\begin{array}{l}\text { Richesse en acides } \\
\text { gras d'intérêt }\end{array}$ & Usage \\
\hline Arachide & $34 \%$ & C18:1 (57 \%) & Peintures \\
\hline Colza & $40-44 \%$ & C18:1 (60 \%) & $\begin{array}{l}\text { Biocarburant, peinture, lubrifiant, agent } \\
\text { anti-poussière }\end{array}$ \\
\hline Colza érucique & $42-44 \%$ & C22:1 (45 \%) & $\begin{array}{l}\text { Lubrifiants, détergents, savons, nylon érucamide } \\
\text { (polyéthylène, peintures...), cosmétiques }\end{array}$ \\
\hline Lin oléagineux & $36-40 \%$ & C18:3 (58 \%) & $\begin{array}{l}\text { Savons, peintures, linoleum, antirouille, mastic, } \\
\text { détergents }\end{array}$ \\
\hline Palme & $20-24 \%$ & C16:0 (40 \%) & Lipochimie (stéarine), travail des métaux (laminage) \\
\hline Olive & $18-22 \%$ & C 18:1 (71\%) & $\begin{array}{l}\text { Lipochimie (oléine), pharmacie, cosmétiques, savons } \\
\text { de toilettes }\end{array}$ \\
\hline Ricin & $50 \%$ & $\mathrm{C} 18: 1 \mathrm{OH}(90 \%)$ & $\begin{array}{l}\text { Peintures, cosmétiques, résines, anticorrosion, } \\
\text { émulsifiant, agent de surface, liquide hydraulique, } \\
\text { lubrifiants de synthèses, polyuréthanes, PVC, nylon } \\
11 \text { ou Rislan B }\end{array}$ \\
\hline Soja & $18-19 \%$ & C18:2 (55 \%) & $\begin{array}{l}\text { Biocarburant, peintures, encres, } \\
\text { lubrifiants, agent de démoulage, agent anti-poussière, } \\
\text { phytosanitaire }\end{array}$ \\
\hline Tournesol & $40-65 \%$ & C18:2 (65 \%) & Biocarburant, peinture \\
\hline Tournesol oléique & & C18:1 (85 \%) & Biocarburant, peinture \\
\hline Tournesol stéarique & & C18:0 (10-20 \%) & Cosmétique \\
\hline
\end{tabular}

Toutefois, les teneurs en huile et la productivité de ce matériel restent encore à améliorer pour une compétitivité accrue.

Le colza érucique vient en seconde position. Il est aujourd'hui cultivé en France sur 15000 ha, principalement dans des bassins de production dédiés (pour éviter les repousses ou les croisements avec le colza conventionnel). Ses usages concernent principalement l'industrie des matières plastiques (érucamides), les détergents et des lubrifiants. Les variétés actuelles, combinées à des bonus attractifs, en font une culture compétitive. La teneur en C22:1 de I'huile plafonne toutefois à $55 \%$ (biologiquement limité à $66 \%$ par la fixation possible uniquement en 2 positions sur $3 \mathrm{du}$ glycérol).

Le colza oléique (qui correspond aussi à du colza bas linoléique) est surtout utilisé aujourd'hui pour l'alimentation en raison des capacités de chauffage accrues pour I'huile (résistance à l'oxydation et absence d'odeurs à la cuisson). Les usages industriels de cette huile s'apparentent à ceux du tournesol oléique. La culture couvre à peine 5000 ha en France. Au vu de son intérêt, cette culture pourrait progresser dans les années à venir.
Le lin oléagineux couvre 10000 ha en France. L'utilisation de la graine entière en alimentation animale est sans nul doute le débouché essentiel pour cette production. Son développement est important au Canada (38\% de la production mondiale) et en Chine (18\%). Les usages industriels concernent principalement la plasturgie (linoléum), les détergents et les peintures. Un regain d'intérêt est noté aujourd'hui pour cette production en France. Les freins essentiels en sont la compétitivité au regard des autres productions.

Le chanvre retrouve quant à lui un intérêt en France, principalement pour la valorisation de sa fibre. Toutefois, la production de graines (chènevis) donne une huile riche en acide linoléique (C18:2) et en alphalinolénique (C18:3). Les applications sont principalement alimentaires, mais aussi cosmétiques.

La cameline est une culture dont l'intérêt à la fois agronomique et industriel est démontré (Bonjean, 1993 ; CETIOM, 1995). Des tentatives d'introduction ont été faites en France dans les systèmes de cultures à la fois avec des types hiver (sans doute plus prometteurs) et des types printemps (Merrien et Chatenet, 1996). Les niveaux de productivité sont au moins équivalents à ceux du colza de printemps et la conduite est plus facile et moins exigeante en intrants. C'est plus une organisation de marché et des débouchés qui font défaut à cette production en France. Elle pourrait retrouver prochainement un intérêt pour son huile utile en cosmétique mais aussi comme source de biocarburant pour moteurs d'avion.

Le ricin a pour sa part fait l'objet de tentatives d'introduction en France dans les années 1990 face à une demande du marché pour les plastiques. L'acide ricinoléique rentre dans la plasturgie (Rilsan), les lubrifiants, les peintures et vernis (Bonjean, 1991 ; Estragnat, 1992 ; CETIOM, 1992). Aujourd'hui, le marché est exclusivement alimenté par des importations (Inde, Chine, Brésil...).

Le crambe d'Abyssinie, comme le ricin, a été l'objet d'études au CETIOM dans les années 1990 (Bureau, 1995, Merrien, 1995, Merrien, 2011). La culture est cependant restée à l'état expérimental et les productions n'ont jamais été significatives. Source alternative d'huile riche en acide érucique, elle n'a jamais été compétitive par rapport au colza érucique. Elle présente en outre un réel handicap au niveau de l'installation 
Tableau 2. Acides gras d'intérêt et usages potentiels pour diverses cultures (Adapté d'après Van Soest, 1993.)

\begin{tabular}{|llll|}
\hline Plante & $\begin{array}{l}\text { Teneur en huile } \\
(\mathbf{\%})\end{array}$ & Richesse en acides gras d'intérêt & Usage \\
\hline Bourrache & $31 \%$ & $\gamma \mathrm{C} 18: 3(31 \%)$ & Cosmétiques \\
\hline Cynara & $25 \%$ & $\mathrm{C} 18: 2(60 \%)$ & Substitut de I'huile de tournesol \\
\hline Cassis & $25 \%$ & $\gamma \mathrm{C} 18: 3(13 \%)$ & Cosmétiques \\
\hline Coriandre & $18-22 \%$ & $\mathrm{C} 18: 1(\mathrm{n}-12)$ & Polymères (nylon), détergents, tensioactifs \\
\hline Jojoba & $45-60 \%$ & $\mathrm{C} 20: 1$ et alcools gras & Cosmétique, huiles de coupe \\
\hline Calendula & $20-21 \%$ & $\mathrm{C} 18: 3$ conj. & Pharmacie, résines, peintures, vernis \\
\hline Cuphea & & $\mathrm{C} 10: 0$ ou C12:0 & Tensioactifs, solvants, émulsifiants \\
\hline Dimorphoteca & $20-26 \%$ & $\mathrm{C} 18: 2$ OH & Polymères, plastiques, peintures et vernis, nylon \\
\hline Vernonia & $38 \%$ & $\mathrm{C} 18: 1-\mathrm{O}($ Epoxy) & Résines, peintures, vernis, plastiques, nylons \\
\hline Crambe & $25-50 \%$ & $\mathrm{C} 22: 1(52 \%)$ & Idem colza érucique \\
\hline Euphorbia lasgascae & $35-49 \%$ & $\mathrm{C} 18: 1-\mathrm{O}($ Epoxy) & Résines, peintures et vernis, plastiques \\
\hline Lesquerella & $20-37 \%$ & $\mathrm{C} 20: 1(52 \%)$ & Résines, peintures et vernis, cosmétique \\
\hline Lunaire & 30 à $40 \%$ & $\mathrm{C} 22: 1(40 \%)$ & Bio-lubrifiants \\
\hline Cameline & $40-42 \%$ & $\mathrm{C} 24: 120 \%$ & Peintures, vernis \\
\hline
\end{tabular}

d'un peuplement régulier, en raison des difficultés de germination des graines (on sème en fait la silicule qui contient une seule graine). Elle reste toutefois une culture peu exigeante en intrants. Le pavot ou cillette est aujourd'hui cultivé principalement en Poitou-Charentes et en Champagne-Ardenne pour la production d'alcaloïdes opiacés sur quelques centaines d'hectares. Les usages des graines sont alimentaires, mais également pharmaceutiques.

\section{Perspectives à I'international}

D'autres cultures oléagineuses peuvent ponctuellement présenter un intérêt, mais la production n'est pas adaptée aux conditions françaises.

Le carthame pousse plutôt dans les régions arides du globe. Ses usages industriels sont décrits en matière de peinture ou de plasturgie. Quelques cultures biologiques sont recensées en vallée du Rhône en raison des vertus diététiques de la graine.

L'onagre est plus réputé pour la richesse de son huile en acide gamma-linolénique, en raison de son intérêt nutritionnel. Les vertus de cette huile sont également valorisées en cosmétique (I'Onagrine ${ }^{\circledR}$ par exemple). L'huile de bourrache offre des applications similaires. Ces deux cultures ont été travaillées au CETIOM et les bases d'un itinéraire technique existent (CETIOM, 1990a et b).

Le limnanthe et le jojoba poussent principalement en Amérique du nord et sont utilisés dans le domaine cosmétique, en raison de la richesse en acide gras à chaîne longue (C20 et C22) de leur huile. En matière industrielle, la résistance de I'huile aux très hautes températures pourrait trouver des applications.

Une société néerlandaise (Calendula Oils) tente de mettre en place une filière de production d'huile de calendula. En raison des faibles rendements de cette culture, les zones de production seraient localisées dans des régions comme le Canada ou l'Europe de l'Est. L'huile de calendula, en raison de ses trois doubles liaisons conjuguées, est très réactive et polymérise facilement.

Parmi les autres plantes candidates à des développements en chimie verte, citons encore l'euphorbe, le cuphéa, lesquerella et vernonia (acide vernolique : C15:1). Le jatropha a également fait l'objet de beaucoup de littérature (plus que de recherches!). Présentée comme plante miracle pour la production de biocarburants dans les régions arides, les études récentes relativisent fortement ses applications (usage local, circuit court pour des biocarburants, savonnerie).

\section{Conclusion}

Plusieurs tentatives d'introduction de cultures oléagineuses à des fins de chimie verte ont été réalisées en France. Toutefois, rares sont les applications industrielles viables de ces cultures. II se pose en effet plusieurs difficultés à leur développement: les ressources génétiques sont parfois difficiles à identifier et il n'existe pas de pools de semences de toutes ces espèces disponibles aisément. Certaines cultures ont fait l'objet d'étude de l'itinéraire technique. Les freins peuvent être au niveau de la densité (crambe) ou encore de la récolte (ricin). Toutefois, ces points sont surmontables, au prix de quelques expérimentations pour la mise au point de I'itinéraire technique. Un atout important pour ces cultures est leur caractère "rustique ", qui permet leur production dans des conditions de faibles niveaux d'intrants (peu d'exigence en fertilisants et peu ou pas de maladies ou de ravageurs). Ce point leur confère ainsi un label de cultures respectueuses de l'environnement, label souvent recherché par les industriels (en cosmétique par exemple). Si les données sont assez cohérentes sur les caractéristiques des graines (teneur en huile, profil d'acides gras...) il est beaucoup plus difficile de disposer d'information sur le réel potentiel de 
rendement de ces productions et les données de la bibliographie sont parfois trompeuses (résultats obtenus en parcelles expérimentales et non dans des conditions de production). Les méthodes d'extraction de l'huile des graines devront aussi faire l'objet d'études (simple pression, paramètre...). Des précautions sont aussi à prévoir pour certaines graines, en raison des risques de toxicité et d'allergie, comme le ricin, l'euphorbe, le jatropha. Plus généralement, c'est donc la compétitivité de ces productions qui, bien souvent, en freine aujourd'hui le développement, au-delà de quelques niches. Une organisation de filière est également essentielle pour ces cultures nouvelles, pour garantir à la fois la disponibilité de la semence, la conduite de la culture et surtout, sa commercialisation à un prix rentable pour le producteur.

\section{RÉFÉRENCES}

Bonjean A. Le ricin une culture pour la chimie fine. Paris : Ed. Golileo/ONIDOL, 1991.

Bonjean A. La cameline-Etude Bibliographique A2P. 1993.

Bureau J. Revue bibliographique sur la culture et les utilisations actuelles et potentielles de 2 cultures nouvelles : le Crambe et le Limnanthès. CR étude ADEME - Poitou-Charentes, 1995.

CETIOM. La culture de Bourrache: fiche technique, 1990a.

CETIOM. La culture d'onagre: fiche technique, $1990 \mathrm{~b}$.

CETIOM. La culture du ricin. Paris : Editions CETIOM, 1992.

CETIOM. Cultures nouvelles et diversifications. Oléoscope 1994 (Suppl. 10).

CETIOM. Alternative oilseed crop Camelina sativa. Report from Contract UE/Air3/CT94/ 2178. 15/12/1995.

Estragnat A. Etude de la croissance et du développement chez le ricin : application au raisonnement de l'impact en situation d'eau limitante. Paris : Editions CETIOM, 1992.

Evrard J. Valorisation industrielle des acides gras des huiles végétales. Paris: Editions CETIOM, 1993.

Mendoza G, Igartua A, Fernandez-Diaz B, Urquiola F, Vivanco S, Arguizoniz R. Vegetable oils as hydraulic fluids for agricultural applications. Grasas y aceites 2011 ; 62 : 2938.

Merrien A. Premiers pas vers un itinéraire technique pour la culture du Crambe. Oléoscope 1995 ; (9) : 21-2.

Merrien A, Chatenet F. Premiers éléments sur la culture de Cameline en France. Dossier Technique CETIOM, juillet 1996.

Merrien A. Crambe and erucic rapeseed: interest and competition. In: CR workshop Crops2Insdustry "Can oilseeds crops offer sustainable alternative for industrial bio-based products ". Bordeaux, Feb 18 ${ }^{\text {th }}, 2011$.

Van Soest, LJM. Potential new oilseed crops for industrial use. Lipid Technology $1993 ;$; : 60-6. 